\title{
Mortalidad intraurbana, envejecimiento y marginación en Hermosillo, Sonora, 2013-2016
}

\author{
Intra-Urban Mortality, Population Ageing and Marginalization \\ in Hermosillo, Sonora, 2013-2016
}

\author{
Pablo Alejandro Reyes-Castro* (iD) https://orcid.org/0000-0002-3091-5530
}

\begin{abstract}
Resumen
El objetivo de este estudio es analizar la distribución espacial intraurbana de la mortalidad por área de residencia y su asociación con condiciones de marginación y envejecimiento poblacional en Hermosillo, Sonora, en el periodo 20I3-20I6. Por medio de información proveniente de certificados de defunción y censal, se realizó un análisis espacial exploratorio de la mortalidad de acuerdo con tres grandes grupos de causas de muerte. El análisis se limitó a la mortalidad y dejó pendiente el componente de morbilidad. Los resultados evidenciaron la fuerte asociación entre la distribución espacial de la mortalidad bruta por enfermedad crónica y el envejecimiento poblacional. Así mismo se evidenció la autosegregación espacial que ejercen los residentes de zonas del nivel más bajo de marginación, quienes tienen mayor capacidad para evitar la muerte por cualquier tipo de causa. Los hallazgos muestran los efectos de la segregación espacial residencial de tipo socioeconómico y demográfico sobre los perfiles de mortalidad en los entornos urbanos.

Palabras clave: mortalidad intraurbana; envejecimiento poblacional; marginación urbana.
\end{abstract}

\begin{abstract}
The aim of this study is to analyze the spatial distribution of intra-urban mortality by area of residence and its association with marginalization and population ageing in Hermosillo, Sonora, during 2013-2016. An exploratory spatial analysis of mortality was performed on three main groups of causes of death by the use of death certificates and census information. The study was limited to mortality and excluded the morbidity component analysis. Results show a strong association between the spatial distribution of chronic disease-related mortality and population ageing; as well as the spatial self-segregation of residents from the lowest level of marginalization areas, which have better conditions to prevent any cause of death. The findings show the effects of residential spatial segregation by socioeconomic and demographic conditions on the mortality profile within urban settings.

Keywords: intra-urban mortality; population ageing; urban marginalization.
\end{abstract}

Cómo citar: Reyes-Castro, P. A. (2019). Mortalidad intraurbana, envejecimiento y marginación en Hermosillo, Sonora, 20I3-20I6. región y sociedad, 31, el209. doi: I0.22198/rys2019/31/I209

* El Colegio de Sonora, Centro de Estudios en Salud y Sociedad. Avenida Obregón 54, Colonia Centro, C.P. 83000. Hermosillo, Sonora, México. Correo electrónico: preyes@colson.edu.mx 


\section{Introducción}

Mucho se ha dicho sobre la transición epidemiológica por la que atraviesa México en su conjunto. Esta transición supone un cambio en el perfil epidemiológico, caracterizado por el predominio de las enfermedades infecciosas, a un patrón en el que las enfermedades crónicas no trasmisibles (ECNT) y las lesiones cobran mayor importancia (Omran, 2005, p. 736). Las razones de este cambio en el perfil de enfermedad encuentran explicación en la serie de cambios demográficos, socioeconómicos, tecnológicos, sociales y culturales, relacionados con el proceso de modernización (Frenk, Bobadilla, Stern, Frejka y Lozano, 1991, p. 450). El descenso de las tasas de fecundidad; la urbanización; la incorporación de la mujer a la fuerza laboral; las mejoras en las condiciones de vivienda, la infraestructura y el saneamiento; así como la implementación de intervenciones preventivas y terapéuticas han impactado en las estructuras de edad y en las expectativas de vida. El resultado ha sido no sólo la reducción en la probabilidad de muerte a edades tempranas sino, además, el envejecimiento paulatino de la población.

Sonora no ha estado exento de estos cambios. Tan sólo durante el período 1990-2010, mientras que las zonas rurales mostraban tasas de crecimiento de $0.1 \%$ anual, las zonas urbanas crecían $2.7 \%$, con una concentración de $71.8 \%$ de la población del estado (Castro Luque, 2015, p. 165). Esta realidad ha sido acompañada por cambios en los perfiles de salud y enfermedad. Durante el mismo período, la proporción de muertes asociadas con enfermedades trasmisibles, maternas, perinatales y nutricionales se redujeron de 19.1 a $10.7 \%$ con respecto al total de defunciones. En contraparte, las defunciones causadas por ECNT cobraron mayor terreno: mostraron un incremento de 65.9 a $76.8 \%$, y la esperanza de vida se incrementó de 72.6 a 76.1 años (Castro Luque, 2015, p. 201; Dirección General de Información en Salud [DGIS-SS], 2017).

Los contextos urbanos, con sus dinámicas de sedentarismo y alimentación, aunados a las exposiciones laborales y a las necesidades de movilización y trasporte, representan hoy día un escenario propicio para el incremento de las ECNT y las lesiones. Sin embargo, resulta poco conveniente dejar de lado aquellas enfermedades infecciosas y las asociadas con el rezago social, ya que los procesos de transición no suelen ser lineales.

La revisión al modelo explicativo de la transición epidemiológica a la luz de condiciones de desigualdad, ayudaron a entender que los procesos de transición no son homogéneos, dando como resultado distintas velocidades e incluso traslapes entre las etapas del proceso y, en consecuencia, en los perfiles de enfermedad entre países y al interior de un mismo país (Frenk et al., 1991, p. 459; Frenk, Lozano y Bobadilla, 1994, p. 91). El traslado de este razonamiento a la escala intraurbana cobra sentido toda vez que los entornos urbanos son también espacios fragmentados donde se expresa la desigualdad (Kroll, Bharucha y Kraas, 2014, p. 4; Lerch, Oris y Wannar, 2017, p. 97). 
La ciudad de Hermosillo, capital del estado de Sonora, constituye el área de estudio de la presente investigación. Según la Encuesta Intercensal 2015 (Instituto Nacional de Estadística y Geografía [INEGI], 2015), cuenta con una población de 819999 habitantes. Aunque es una localidad con índices muy bajos de rezago y de marginación social en comparación con el contexto nacional, muestra en su interior diferencias socioeconómicas y de estados de vivienda que dan cuenta de las condiciones de desigualdad (véase figura 1). Por otra parte, el proceso de crecimiento de la ciudad refleja, a su vez, procesos de envejecimiento poblacional disímiles, lo que hace suponer distintos perfiles de enfermedad según las estructuras poblacionales en su interior $\mathrm{y}$, por lo tanto, distintas necesidades según las características demográficas de la población (véase figura 1).

Figura 1. Marginación urbana y razón de dependencia de vejez en Hermosillo, 2010




grandes grupos de causas de muerte, a saber: grupo I. enfermedades trasmisibles, maternas, perinatales y nutricionales; grupo II. enfermedades crónicas no trasmisibles (ECNT); y grupo III. causas externas.

El objetivo central de esta investigación consiste en realizar un análisis espacial exploratorio para determinar la distribución intraurbana de la mortalidad en cada uno de los tres grandes grupos de causas y su asociación con el envejecimiento poblacional y la marginación urbana. Para situar el análisis propuesto en el contexto epidemiológico actual que vive la ciudad en su conjunto, se planteó como objetivo secundario estimar la carga de la mortalidad ocurrida en Hermosillo en el período 2013-2016 mediante el indicador de años de vida perdidos (AVP) atribuida a los tres grandes grupos de causas de muerte mencionados.

\section{Métodos}

Fuentes de información

Para abordar los objetivos planteados se utilizaron dos tipos de fuentes. La información de mortalidad se obtuvo mediante el acceso directo a la base de datos de la Secretaría de Salud del Estado de Sonora, compuesta por 15106 certificados de defunción correspondientes al período 2013-2016. Dada la necesidad de contar con información del lugar de residencia, el Comité de Bioética del Departamento de Medicina y Ciencias de la Salud, de la Universidad de Sonora, previamente revisó y aprobó el proyecto "Perfiles de mortalidad intra-urbana por área de residencia en tres ciudades del Estado de Sonora" (dictamen DMCS/ CBIDMCS/D-100), aprobado el día 3 de octubre de 2017). A partir de la variable "causa básica de la defunción", se categorizaron las defunciones según los criterios establecidos por la lista Global Burden of Disease (GBD) 165 (DGIS-SS, 2017). La categorización se realizó para el primer nivel de agrupación que define los grupos I, II y III antes mencionados y se incluyó la desagregación hasta un segundo nivel para posibilitar las descripciones de causas más específicas en cada uno de los grupos, según las necesidades de análisis (véase tabla 1).

La información demográfica se obtuvo del Censo de Población y Vivienda 2010 (INEGI, 2010b), por tratarse de la fuente de información más próxima al período de estudio con información a nivel de área geoestadística básica (AGEB). Por su parte, la información de marginación se obtuvo del CONAPO (2010), el cual construye el índice de marginación urbana (IMU) a partir de información poblacional y de vivienda proveniente del mismo censo.

El IMU se integra por diez variables: porcentaje de población de 6 a 14 años que no asiste a la escuela, porcentaje de población de 15 años o más sin educación básica completa, número de hijos nacidos muertos, porcentaje de población sin derechohabiencia a servicios de salud, porcentaje de viviendas sin conexión de agua, sin drenaje, sin WC, sin refrigerador, con piso de tierra y condiciones de hacinamiento. 
Tabla 1. Categorías de causas GBD, primer y segundo niveles

\begin{tabular}{|c|c|c|}
\hline Primer nivel & Segundo nivel & Código CIE-10 \\
\hline $\begin{array}{l}\text { I. Enfermedades } \\
\text { trasmisibles, } \\
\text { maternas, } \\
\text { perinatales y } \\
\text { nutricionales. }\end{array}$ & $\begin{array}{l}\text { A. Enfermedades infecciosas y parasitarias. } \\
\text { B. Infecciones respiratorias. } \\
\text { C. Causas maternas. } \\
\text { D. Ciertas afecciones originadas } \\
\text { E. en el periodo perinatal. } \\
\text { F. Deficiencias de la nutrición. }\end{array}$ & $\begin{array}{c}\text { A00-B99, D50-D53, } \\
\text { D64.9, E00-E02, } \\
\text { E40-E46, E50, E51-E64, } \\
\text { G00-G04, H65-H66, } \\
\text { J00-J06, J09-J18, } \\
\text { J20-J22, N70-N73, } \\
\text { O00-O99, P00-P96 }\end{array}$ \\
\hline $\begin{array}{l}\text { II. Enfermedades } \\
\text { no trasmisibles. }\end{array}$ & $\begin{array}{c}\text { A. Tumores malignos. } \\
\text { B. Otros tumores. } \\
\text { C. Diabetes mellitus. } \\
\text { D. Enfermedades endocrinas, } \\
\text { E. metabólicas, hematológicas } \\
\text { F. e inmunológicas. } \\
\text { G. Trastornos mentales y enfermedades } \\
\text { H. del sistema nervioso. } \\
\text { I. Enfermedades de los órganos } \\
\text { J. de los sentidos. } \\
\text { K. Enfermedades cardiovasculares. } \\
\text { L. Enfermedades respiratorias. } \\
\text { M. Enfermedades digestivas. } \\
\text { N. Enfermedades del sistema genitourinario. } \\
\text { O. Enfermedades de la piel. } \\
\text { P. Enfermedades del sistema } \\
\text { Q. músculo-esquelético. } \\
\text { R. Anomalías congénitas. } \\
\text { S. Enfermedades de la boca. }\end{array}$ & $\begin{array}{c}\text { C00-C97, D00-D48, } \\
\text { D55-D64 (salvo D 64.9), } \\
\text { D65-D89, E03-E07, } \\
\text { E10-E16, E20-E34, } \\
\text { E65-E88, F01-F99, } \\
\text { G06-G98, H00-H61, } \\
\text { H68-H93, I00-199, } \\
\text { J30-J98, K00-K92, } \\
\text { L00-L98, M00-M99, } \\
\text { N00-N64, N75-N98, } \\
\text { O00-Q99 }\end{array}$ \\
\hline $\begin{array}{l}\text { III. Causas externas } \\
\text { de morbilidad y } \\
\text { mortalidad. }\end{array}$ & $\begin{array}{l}\text { A. Accidentes. } \\
\text { B. Lesiones intencionales } \\
\text { C. (homicidios y suicidios). } \\
\text { D. Eventos (lesiones) de intención } \\
\text { E. no determinada. }\end{array}$ & V01-Y89 \\
\hline $\begin{array}{l}\text { IV. Causas mal } \\
\text { definidas. }\end{array}$ & 0. Causas mal definidas. & R00-R99 \\
\hline
\end{tabular}

Fuente: elaboración propia con base en información de la Dirección General de Información en Salud (DGIS-SS, 2017).

\section{Georreferenciación}

Se georreferenció, a nivel de AGEB, $97.6 \%$ del total de defunciones ocurridas en el período de estudio. ${ }^{2}$ De origen, el certificado de defunción utiliza la colonia como el área mínima de ubicación de residencia. Por lo tanto, se requirió resolver la discrepancia entre unidades geográficas (AGEB versus colonia) mediante un procedimiento de normalización geométrica en ArcGIS 10.1, el cual consiste en redistribuir en número de casos registrados por colonia de forma proporcional entre la(s) AGEB que la(s) intersecan.

2 Se seleccionó el período 2013-2016 por su factibilidad metodológica. En 2012, se realizaron ligeros cambios al certificado de defunción, entre los cuales se incluyó un campo específico para la variable "colonia" de residencia. Se aprovechó este cambio reflejado a partir de 2013 para georreferenciar un número considerable de defunciones de forma más rápida y eficiente agrupando los datos a pequeña escala. 
Una vez concluido este procedimiento, se estimaron tasas brutas de mortalidad (TBM) y tasas de mortalidad ajustadas por edad (TMA) a nivel de AGEB para los tres grupos de causas de muerte. El ajuste de tasas se realizó mediante método directo utilizando la población de Hermosillo de 2015 como referencia. ${ }^{3}$ Las tasas se presentan como defunciones por cada 10 mil habitantes. Por último, las tasas se suavizaron mediante el método de Bayes empírico para corregir la inestabilidad de varianzas de aquellas AGEB pequeñas con poca población (Besag, York y Mollié, 1991, p. 6).

Cálculo de años de vida perdidos (AVP)

En primer lugar, se estimó la carga de la mortalidad atribuida a los tres grupos de causas de muerte para la ciudad en su conjunto mediante el indicador de años de vida perdidos (AVP), el cual resulta de la suma de los productos del número de muertes por una causa específica de cada edad por la esperanza de vida a la edad de muerte (Martinez, Soliz, Caixeta y Ordunez, 2019). La fórmula es la siguiente:

$$
A V P=\sum_{0}^{l} d_{x} \mathrm{X} e_{x}
$$

Donde:

$d_{x}=$ número de defunciones a la edad $x$.

$e_{x}=$ esperanza de vida estándar a la edad $x$.

$l=$ último grupo de edad.

Para el cálculo se utilizó la tabla de esperanza de vida por edad estimadas por el GBD Project (Institute of Health Metrics and Evaluation, 2017). Una vez obtenidos los AVP para cada grupo de causas, se calculó la proporción con respecto al total de AVP.

\section{Análisis espacial exploratorio intraurbano}

Se realizó un análisis espacial exploratorio sobre la distribución espacial de la TMB y la TMA. Por un lado, se exploró la autocorrelación espacial global univariada con el software GeoDa 1.10.0.8, utilizando el índice de Moran global (I) para determinar si la mortalidad correspondiente a cada uno de los tres grupos de causas se distribuía de forma aleatoria o si, por el contrario, la distribución espacial tendía a agruparse de acuerdo con valores de mortalidad similares o disímiles a través de la ciudad (Anselin, 1996, p. 116). La interpretación del I de Moran se hace a partir de los valores posibles de autocorrelación espacial que van de 1 (dispersión perfecta) a 1 (agrupamiento perfecto).

3 El sistema para la consulta de información censal (SCINCE) 2010 del INEGI no presenta la información de población por grupos quinquenales, por lo que se estandarizaron las tasas utilizando los grupos de edad reportados: 0-4, 5-14, 15-29, 30-49, 50-59, 60 y más. 
El índice de Moran global queda definido de la siguiente forma:

$$
I=\frac{n}{S_{0}} \frac{\sum_{i} \sum_{j} w_{i, j} z_{i} z_{j}}{\sum_{i} z_{i}^{2}}
$$

Donde:

$n=$ número total de unidades espaciales (AGEB).

$z_{i}=$ desviación de la unidad espacial $i$ con respecto a su media $\left(\mathbf{x}_{i}-\overline{\mathbf{x}}\right)$.

$w_{i, j}=$ peso espacial entre AGEB $i$ y $j$.

$S_{0}=$ sumatoria de todos los pesos espaciales. ${ }^{4}$

Dado que el agrupamiento de la mortalidad podría no expresarse de forma global en la ciudad sino en zonas específicas, se realizó también un análisis de indicadores locales de asociación espacial (local indicators of spatial association, LISA, por sus siglas en inglés) para la detección y mapeo de agrupamientos de AGEB de alta mortalidad, AGEB de baja mortalidad, o AGEB atípicos (de alta mortalidad con áreas vecinas de baja mortalidad, y viceversa) (Anselin, 1995, p. 94). El indicador local de autocorrelación espacial se estima así:

Donde:

$$
I_{i}=\left(z_{i} / m_{2}\right) \sum_{j=1}^{n} w_{i j} Z
$$

$m_{2}=$ varianza.

$\mathbf{Z}=$ desviaciones de la media $\left(\boldsymbol{x}_{i}-\overline{\boldsymbol{x}}\right)$.

$w_{i, j}=$ peso espacial entre AGEB $i$ y $j$.

Por último, se analizó la asociación espacial bivariada entre la TMB de cada uno de los tres grupos de causas de muerte con la razón de dependencia de vejez (población de 65 años o más por cada 100 personas de 15 a 64 años), utilizando de nueva cuenta el índice de Moran global y el análisis de LISA pero en su versión bivariada para detectar agrupamientos de AGEB con alta TBM/alto envejecimiento, baja TBM/bajo envejecimiento, y valores atípicos. El mismo procedimiento se utilizó para asociar la TMA y el IMU. La significancia estadística de los resultados se consideró tomando como criterio un valor de $\mathrm{p}<0.05$.

\section{Resultados}

Los años de vida perdidos (AVP)

Previo al análisis de la distribución intraurbana de los tres grupos de causas de muerte, fue de interés dimensionar cada una de estas causas en términos de

4 Los pesos espaciales se generaron mediante una matriz de pesos espaciales tipo torre (rook) de primer orden que determina la contigüidad (vecindad) entre áreas. 
la pérdida de años de vida que representan para la ciudad en su conjunto y así contar con una referencia del perfil epidemiológico actual en la localidad.

En la tabla 2 se muestran los AVP estimados para el período de estudio. Según los resultados obtenidos, la población de Hermosillo perdió un total de 440142 años de vida; son los hombres la población sobre la que recayó en mayor medida la carga de la mortalidad (hombres $=61.6 \%$ versus mujeres $=38.4 \%$ ). Sólo $16.3 \%$ de la carga de la mortalidad ocurrida en Hermosillo se atribuyó a enfermedades del tipo infeccioso, materno, perinatal y nutricional (grupo I); recayó 38.4\% de dicha carga sobre la población infantil menor a 1 año (véase figura 2). En contraparte, las ECNT (grupo II) fueron responsables de $68.3 \%$ de la pérdida de años en la población, carga principalmente atribuida a enfermedades cardiovasculares, tumores malignos, diabetes mellitus y enfermedades digestivas; se concentró cerca de la mitad (47.8\%) de los años perdidos en el grupo de 60 años o más (véase figura 2). Respecto de las muertes por causas externas, éstas aportaron 14.1\% de los AVP. Al desagregar por sexo, la proporción de estas causas fue tres veces mayor en los hombres que en las mujeres (19.0\% versus $6.4 \%)$. Las causas específicas correspondieron en mayor medida a accidentes relacionados con el tránsito, seguidos por los homicidios.

A manera de síntesis, tres aspectos llaman a la reflexión. Por una parte, las condiciones socioeconómicas del entorno urbano que permiten evitar la enfermedad temprana asociada con enfermedades del grupo I que, si bien persistieron en su afectación principalmente entre niños menores de un año, representaron una proporción bastante menor en comparación con las ECNT. Por otra parte, fue evidente el predominio de la mortalidad por ECNT, que ejercen su mayor carga en edades avanzadas. Por último, y en medio de la dicotomía agudo-infeccioso versus crónico-no trasmisible, se encontró la carga de las muertes por causas externas, donde los accidentes y homicidios presentan los retos en materia de seguridad y salud pública asociados con la movilidad urbana, el crimen y la convivencia social en la ciudad.

La mortalidad y el envejecimiento poblacional en la ciudad: las tasas brutas

A la par del incremento de las ECNT, la ciudad de Hermosillo sigue sufriendo cambios en su estructura de edad y en el proceso de envejecimiento de su población. Entre 1990 y 2010, la razón de dependencia de vejez pasó de 5.8 a 7.6 personas de 65 años o más por cada 100 personas en edad productiva (15 a 64 años) (INEGI, 2010a). Sin embargo, tal como se planteó en la introducción, el envejecimiento poblacional no es homogéneo en la ciudad, de tal suerte que, mientras en las AGEB localizadas en zonas céntricas la razón de dependencia rondó entre 12.9 y 35.6 , los asentamientos localizados en la periferia mostraron razones entre 0 y 1.5. En la tabla 3 se muestran los valores de la autocorrelación espacial global de las variables de estudio. En el caso de la razón de dependencia de vejez, es evidente la fuerte agrupación espacial de la población adulta mayor $(l=0.8343)$.

Por otra parte, los resultados de autocorrelación espacial univariada mostrados en la misma tabla 3 , dan cuenta del grado de dependencia espacial de la 
Reyes-Castro



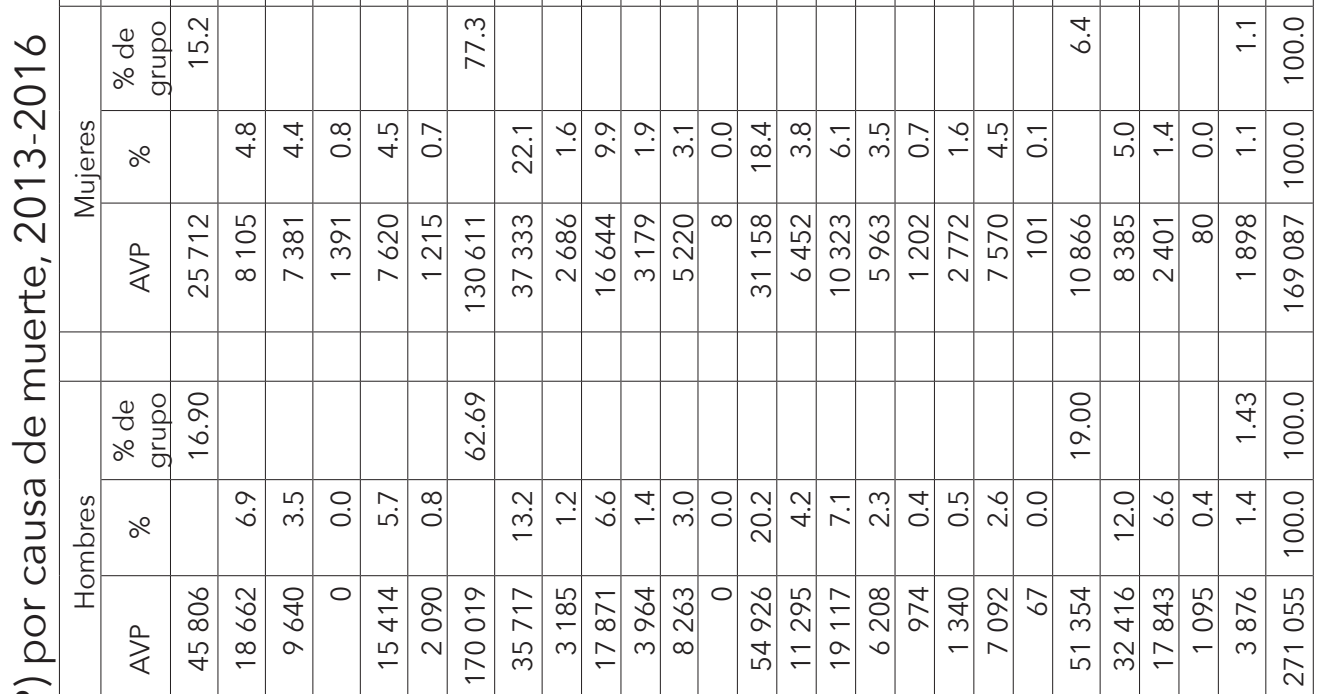






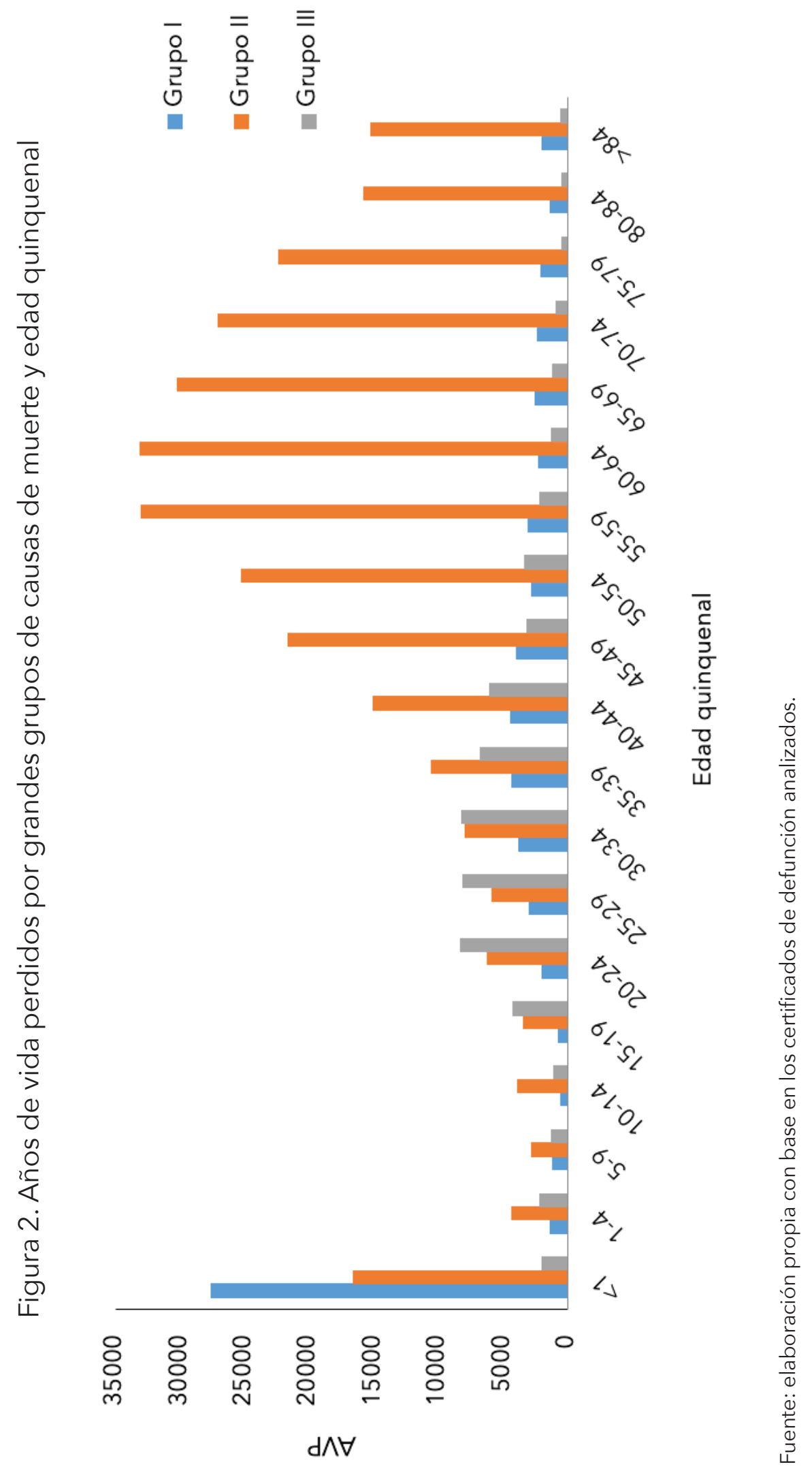


mortalidad correspondiente a cada uno de los tres grupos de causas de muerte. De entre ellas, la mortalidad por ECNT (grupo II) tendió a agruparse de manera más fuerte $(l=0.660)$, seguida por una autocorrelación moderada de la mortalidad relacionada con enfermedades del grupo I $(l=0.4705)$ y, por último, un patrón de agrupamiento mucho más débil por parte de las muertes por causas externas $(l=0.2303)$.

Tabla 3. Análisis de autocorrelación global univariado y bivariado, tasas crudas

\begin{tabular}{|c|c|c|c|}
\hline $\begin{array}{l}\text { Grupo de causas de muerte } \\
\text { y condición sociodemográfica }\end{array}$ & $\begin{array}{l}\text { I de Moran } \\
\text { univariado }\end{array}$ & $\begin{array}{l}\text { I de Moran } \\
\text { bivariado } \\
\text { (TBM-razón de } \\
\text { dependencia) }\end{array}$ & $\begin{array}{c}\text { I de Moran } \\
\text { bivariado } \\
\text { (TBM-marginación) }\end{array}$ \\
\hline \multicolumn{4}{|l|}{ Mortalidad } \\
\hline $\begin{array}{c}\text { Grupo I. Infecciosas, maternas, } \\
\text { perinatales } \\
\text { y nutricionales. }\end{array}$ & $0.4705^{\star \star \star}$ & $0.4989^{\star \star \star}$ & $0.0510^{\star}$ \\
\hline $\begin{array}{l}\text { Grupo II. Enfermedades } \\
\text { crónicas no trasmisibles. }\end{array}$ & $0.6602^{\star \star \star}$ & $0.7209^{\star \star \star}$ & $0.0741^{* *}$ \\
\hline Grupo III. Causas externas. & $0.2303^{\star \star \star}$ & $0.2966^{\star \star \star}$ & $0.1149^{* *}$ \\
\hline \multicolumn{4}{|l|}{ Condición socio-demográfica } \\
\hline Razón de dependencia de vejez. & $0.8343^{\star \star \star}$ & --------- & --------- \\
\hline Marginación urbana. & $0.6010^{\star \star \star}$ & --------- & --------- \\
\hline${ }^{*} p<0.05,{ }^{* *} p<0.01, p<0.001$ & & & \\
\hline
\end{tabular}

Fuente: elaboración propia con base en los certificados de defunción analizados y el |NEG| (2010b).

Los patrones locales de estos agrupamientos (LISA) se muestran en las figuras $3 d$, 3e y $3 f$, donde se observa con claridad una zona compacta en el centro de la ciudad conformada por AGEB con altas TBM atribuidas a ECNT (grupo II), mientras que las áreas con tasas más bajas tienden a agruparse en las periferias (véase figura 3e). Por su parte, si bien la mortalidad correspondiente al grupo I sigue también esta progresión de centro a periferia, la zona central compacta de alta mortalidad antes mencionada resulta más dispersa para este grupo de causas (véase figura 3d). En cuanto a la mortalidad por causas externas (grupo III), ésta deja de seguir el patrón antes mencionado para mostrar un agrupamiento de alta mortalidad en el lado este de la ciudad y agrupamientos mixtos de alta y alta-baja mortalidad distribuidos en distintos puntos de la ciudad (véase figura 3 f). 
Figura 3. Mortalidad bruta y agrupamientos locales por grandes grupos de causas, 2013-2016

Tasas bruta de mortalidad (con suavizamiento Bayes empírico)

Figura 3a. Mortalidad grupo I $\quad$ Figura 3b. Mortalidad grupo II

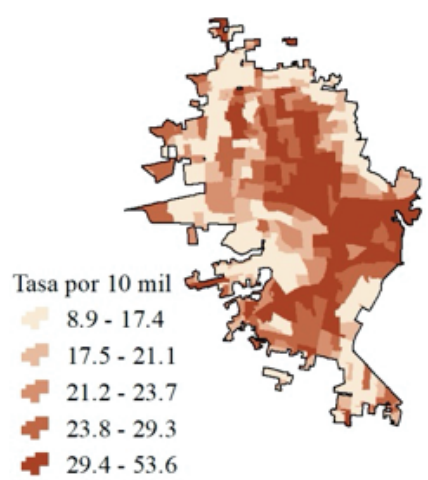

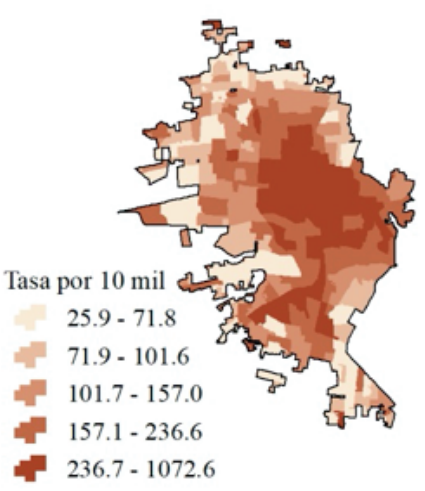

Figura 3c. Mortalidad grupo III



Agrupamientos locales univariados (tasa bruta de mortalidad)

Figura3d. Agrupamientos grupo I Figura3e. Agrupamientos grupo II

Figura3f. Mortalidad grupo III



Agrupamientos locales bivariados (tasa bruta de mortalidad-envejecimiento)



Figura3g. Agrupamientos grupo I Figura3h. Agrupamientos grupo II
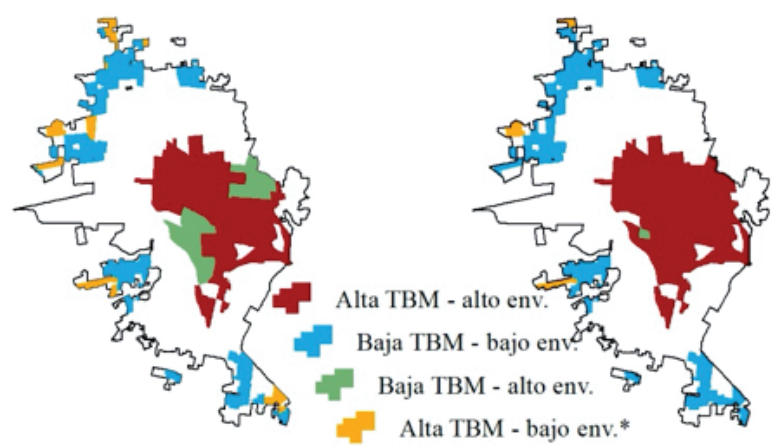

*env.: envejecimiento
Figura3i. Mortalidad grupo III

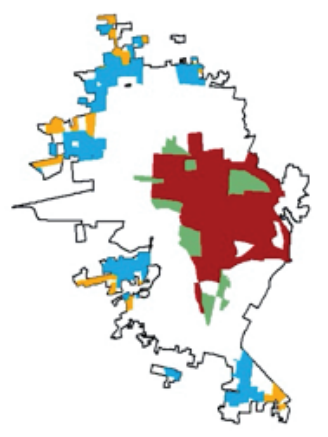

Fuente: elaboración propia con base en los certificados de defunción analizados y el INEGI (2010b). 
Se observó una fuerte autocorrelación espacial global bivariada $(I=0.7209)$ entre la TBM por ECNT correspondiente al grupo II y la razón de dependencia de vejez (véase tabla 3). El centro de la ciudad presentó una zona compacta de agrupamiento de alta TBM/alto envejecimiento, mientras que la periferia mostró el patrón contrario, es decir, agrupamientos de baja TBM/bajo envejecimiento (véase figura $3 \mathrm{~h}$ ).

En resumen, la agrupación espacial del envejecimiento en la zona centro de la ciudad se vio fuertemente asociada con la TBM por ECNT, correspondiente al grupo II, y sólo de forma moderada con el grupo I. Esta distribución epidemiológica y demográfica responde en mayor medida a los patrones de localización/ movilización residencial de la población en la ciudad (Garrocho y Campos-Alanis, 2015, p. 116; Moore y Pacey, 2004, p. s6). La distribución de la población adulta mayor en la ciudad de Hermosillo presenta una organización espacial concéntrica que ha persistido en las últimas décadas (Álvarez de la Torre, 2017, p. 168). Como se esperaba, la mayor concentración de adultos mayores evidenció el incremento en las probabilidades de muerte en AGEB localizadas en dicha zona, incluyendo, por supuesto, la muerte cardiovascular por causa natural.

Este patrón de concentración espacial de la población adulta mayor no es privativo de la ciudad de Hermosillo. Se ha observado esta distribución en diversas ciudades medias mexicanas, independientemente del crecimiento morfológico de la mancha urbana, y forma parte de un fenómeno de segregación socioespacial residencial que poco a poco cobra atención en el país (Garrocho y Campos-Alanis, 2016, p. 104; Álvarez de la Torre, 2017, p. 174). Por lo tanto, cabe suponer un comportamiento similar de la distribución espacial de la TBM por ECNT en ciudades similares de tamaño medio.

Los motivos de segregación socioespacial residencial del adulto mayor no necesariamente tienen orígenes negativos, ya que las decisiones con respecto a la localización/movilidad residencial pueden tener carácter voluntario asociado al arraigo, la presencia de redes familiares y vecinales de apoyo y al acceso a bienes y servicios. Más allá de los motivos, en lo que respecta a la perspectiva de la mortalidad abordada en este estudio, ${ }^{5}$ el conocimiento del perfil epidemiológico y de las estructuras de edad intraurbana puede ayudar a la toma de decisiones referentes al ambiente construido con fines de mejorar la salud de este sector poblacional.

Diversos estudios urbanos con población adulta mayor han evidenciado un efecto protector ante la prevalencia y el riesgo de muerte por enfermedad cardiovascular entre aquellos residentes de lugares con mayor cantidad de áreas verdes, independientemente del nivel socioeconómico individual o del vecindario (Massa, Pabayo, Lebrão y Filho, 2016, p. 6; Wang, Lau, Yu, Wong, Kwok y Woo, 2017, p. 5). Así mismo, se ha reportado mayor riesgo de muerte en adultos mayores con poca disponibilidad a comercios de comida saludable, sobre todo entre aquellos que no utilizan automóvil (Tani, Suzuki, Fujiwara, Hanazato, Kondo, Miyaguni y Kondo, 2018, p. 1).

5 En el análisis realizado, sólo se consideró el componente de la mortalidad, pues es una realidad la emergencia de ECNT a edades cada vez más tempranas. Por lo tanto, la valoración de prevalencias de ECNT y sus factores de riesgo en el ámbito intraurbano es un asunto pendiente para futuros estudios. 
Por otra parte, los resultados son útiles para orientar mejoras en el acceso y la administración de los recursos de salud en la ciudad (Cagney, 2019, p. 64; Diez-Roux, 2003, p. 583; Murray y Grubesic, 2015, p. 244). Los perfiles obtenidos permiten identificar a los residentes de aquellas zonas que presumiblemente demandarán en mayor medida servicios de salud para la atención de padecimientos crónicos y suplir dichas necesidades de acceso. Futuras investigaciones pueden contrastar estos resultados con el acceso geográfico y efectivo que en la actualidad brindan los servicios de salud a la población adulta mayor.

Por lo pronto, es posible suponer mejores condiciones de acceso a servicios de salud en relación con otras ciudades medias ubicadas en entidades de mayor marginación. Por ejemplo, de acuerdo con datos obtenidos por encuesta, 63.3\% de los jefes de hogar mayores de 65 años o más en Hermosillo consideran contar con hospitales, clínicas y centros de salud cercanos, dato que contrasta con la realidad de una ciudad como Tuxtla Gutiérrez, donde sólo $34.9 \%$ percibe cercanos los servicios de salud (estimación propia a partir de INEGI, 2014). Diferencias socioeconómicas en la derechohabiencia, así como en las capacidades de movilización y trasporte son importantes aspectos a considerar para una mejor comprensión de la relación que guardan el acceso geográfico a los servicios y su impacto sobre la salud al comparar estos resultados con otras ciudades del país.

Ahora bien, los resultados también indicaron que, en términos brutos, estas zonas céntricas envejecidas y de altas TBM por ECNT (e incluso por enfermedades del grupo I asociadas comúnmente al rezago social) no fueron precisamente las más marginadas de la ciudad. Los resultados mostraron una débil asociación entre las TBM y la marginación urbana (véase tabla 3). En otras palabras, el envejecimiento poblacional de la ciudad, aun cuando conlleva una mayor carga de muerte, entre otras cosas por la proximidad a los límites biológicos de la vida humana, no representó por sí solo un sinónimo de vulnerabilidad socioeconómica. En este sentido, si bien las TBM observadas permitieron reflejar la realidad con respecto a los diferentes grados de envejecimiento en la ciudad, las diferentes estructuras de edad presentaron un potencial efecto confusor al tratar de evidenciar la asociación entre la mortalidad y la marginación (Robson, Purdie, Cram y Simmonds, 2007, p. 8). En el siguiente apartado se discute la distribución de la mortalidad después de remover el sesgo inducido por las estructuras de edad.

Mortalidad, marginación y zonas de protección: las tasas ajustadas

Cuando se habla de envejecimiento, debe recalcarse que se hace en su sentido poblacional. Si bien el análisis de las TBM mostró una débil asociación con la marginación urbana (véase tabla 3), ello no implica que las personas adultas mayores de zonas marginadas tengan mejores condiciones de salud. Se trata más bien de un asunto de baja proporcionalidad con respecto al total de habitantes en su área de residencia. Como se mencionó antes, las tasas brutas están sesgadas por las diferentes estructuras de edad y, en consecuencia, por los distintos grados de envejecimiento. Una vez controlado el efecto confusor de la edad mediante la estandarización de tasas, fue posible explorar la asociación de la TMA y la marginación urbana, partiendo del supuesto de que las poblaciones de todas las AGEB compartían la misma estructura de edad. 
Los resultados del análisis de autocorrelación espacial global univariada mostraron una débil autocorrelación de la mortalidad de los tres grupos considerando la ciudad como un todo (véase tabla 4). La mortalidad correspondiente al grupo I fue la que presentó los valores mayores $(l=0.3127)$ pero aun así resultaron débiles. Los resultados del análisis de autocorrelación espacial global bivariada también mostraron una débil autocorrelación entre el IMU y los tres grupos de causas de muerte con valores de $I<0.2$ (véase tabla 4). Es decir, la distribución espacial de la TMA guardó poca relación con las condiciones de marginación intraurbana de las AGEB de residencia, al menos en los términos generales que plantea el índice de Moran global.

Tabla 4. Análisis de autocorrelación global univariado y bivariado, tasas ajustadas por edad

\begin{tabular}{|c|c|c|}
\hline Grupo de causa de muerte & I de Moran univariado & $\begin{array}{l}\text { I de Moran bivariado } \\
\text { (TMA-marginación) }\end{array}$ \\
\hline \multicolumn{3}{|l|}{ Mortalidad } \\
\hline $\begin{array}{l}\text { Grupo I. Infecciosas, maternas, } \\
\text { perinatales y nutricionales. }\end{array}$ & $0.3127^{\star \star \star}$ & $0.1638^{\star \star \star}$ \\
\hline $\begin{array}{l}\text { Grupo II. Enfermedades } \\
\text { crónicas no trasmisibles. }\end{array}$ & $0.2319^{\star \star \star}$ & $0.1091^{\star \star \star}$ \\
\hline Grupo III. Causas externas. & $0.1631^{\star \star \star}$ & $0.1607^{\star \star \star}$ \\
\hline \multicolumn{3}{|l|}{ Condición socio-demográfica } \\
\hline Marginación urbana. & $0.6010^{\star \star \star}$ & --------- \\
\hline${ }^{*} p<0.05,{ }^{* \star} p<0.01,{ }^{* \star *} p<0.001$ & & \\
\hline
\end{tabular}

Fuente: elaboración propia con base en los certificados de defunción analizados y el INEG| (2010b).

Sin embargo, aun cuando el agrupamiento resultó débil a nivel global, fue posible detectar zonas específicas de agrupamiento. Los resultados del análisis de LISA bivariado mostraron pequeños agrupamientos locales en la periferia de la ciudad correspondientes a zonas vulnerables con alta TMA/alta marginación. Los tres grupos de causas de muerte fueron consistentes en la presencia de agrupamientos de este tipo en la periferia suroeste de la ciudad, mientras que la periferia oriente mostró un agrupamiento asociado a muertes del grupo III correspondientes a causas externas (véanse figuras $4 \mathrm{~g}, 4 \mathrm{~h}$ y $4 \mathrm{i}$ ). Por otro lado, los resultados mostraron dos amplias zonas de agrupamientos de baja TMA/baja marginación, que conforman un eje que va del centro al norte, y un segundo eje, del centro al lado oeste de la ciudad. Ambas zonas de agrupamiento de baja TMA se observaron en los tres grupos de causas, con lo que se reflejan las ventajas socioeconómicas de estos residentes con respecto al resto (véanse figuras $4 \mathrm{~g}, 4 \mathrm{~h}$ y $4 \mathrm{i})$. 
Figura 4. Mortalidad ajustada y agrupamientos locales por grandes grupos de causas, 2013-2016

Tasas de mortalidad ajustada por edad (con suavizamiento Bayes empírico)

Figura 4a. Mortalidad grupo I


- $25.9-30.8$

$30.9-76.0$
Figura 4b. Mortalidad grupo II

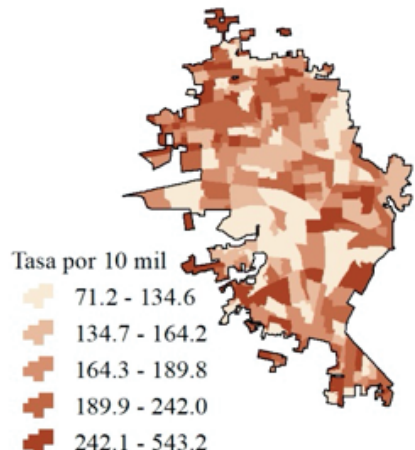

Figura 4c. Mortalidad grupo III

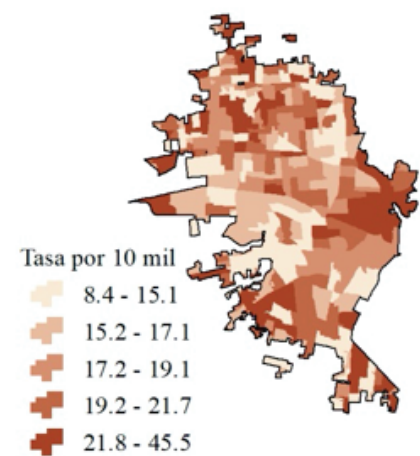

Agrupamientos locales univariados (tasa de mortalidad ajustada por edad)

Figura 4d. Agrupamientos grupo I Figura 4e. Agrupamientos grupo II

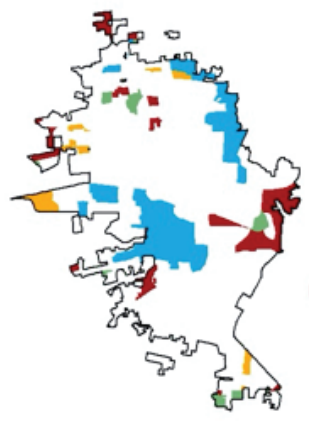

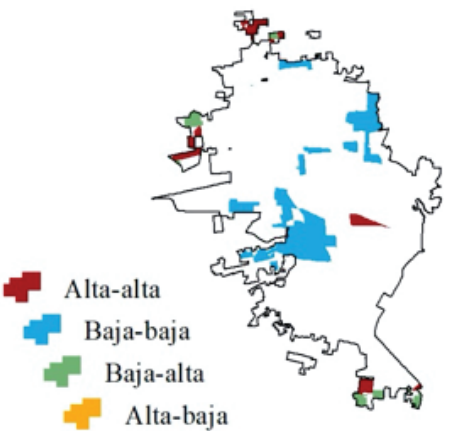

Figura 4f. Mortalidad grupo III

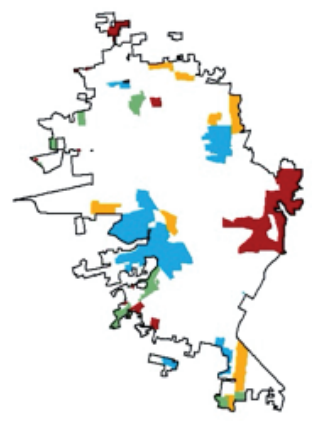

Agrupamientos locales bivariados (tasa de mortalidad ajustada por edad-marginación)

Figura 4g. Agrupamientos grupo I Figura 4h. Agrupamientos grupo II



Figura 4i. Mortalidad grupo III

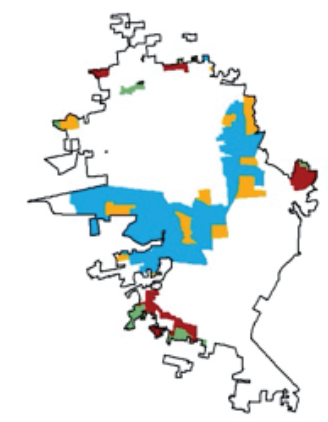

Fuente: elaboración propia con base en los certificados de defunción analizados y el INEGI (2010b). 
Tres aspectos pueden destacarse de estos resultados. Primero, una de las utilidades del análisis exploratorio consiste en detectar aquellos espacios urbanos vulnerables de acuerdo con sus características socioeconómicas y de salud con fines de focalizar intervenciones. En este estudio fue posible detectar zonas segregadas en la periferia con condiciones de alta TMA y marginación. Como antecedente de estas zonas, estudios previos han encontrado asociaciones entre niveles altos de mortalidad infantil y condiciones de alta marginación urbana y vulnerabilidad socio-ambiental (Álvarez et al., 2009, p. 34; Lara-Valencia et al., 2012, p. 372).

Más aún, la eliminación del efecto de la edad permitió ver que el impacto de la mortalidad en estas zonas marginadas no se restringió a causas usualmente asociadas al rezago social con impactos considerables a edades tempranas, sino también con respecto a ECNT y causas externas. Éste es un patrón ya observado en ciudades de países en vías de desarrollo cuyas áreas más marginadas tienden a presentar lo que se ha denominado la doble carga de la enfermedad, es decir, la presencia de altas incidencias y riesgo de muerte por enfermedades infecciosas, que coexisten con un incremento en las prevalencias y riesgo de muerte por ECNT (Agyei-Mensah y de-Graft Aikins, 2010, p. 893; Kroll et al., 2014, p. 3; Snyder, Costa, Lima, Calcagno, Couto, Riley, Reis, Ko, y Ribeiro, 2017, p. 7). Las intervenciones de los tomadores de decisiones locales en áreas vulnerables necesitan mejorar el acceso y la atención de los servicios de salud, así como las condiciones de vida inmediatas.

En segundo lugar, es importante contextualizar estos resultados. Un entendimiento amplio de las desigualdades de la salud intraurbana no puede desligarse de aquellos determinantes distales de mayor escala (Brisbois, O'Campo y Kamalanathan, 2019, p. 77). En ese sentido, dentro del contexto nacional, Hermosillo cuenta con bajos niveles de marginación. Si bien resultó relevante identificar estas zonas vulnerables de alta TMA que corresponden a áreas conocidas por su condición de segregación espacial debido a motivos socioeconómicos (Consejo Nacional de Evaluación de la Política de Desarrollo Social [CONEVAL], 2010), en realidad representan un número pequeño en comparación con la totalidad de Hermosillo. Mientras que casi la mitad (48\%) de las AGEB se categorizaron con niveles muy bajos de marginación, tan sólo $9.8 \%$ se identificaron con nivel alto y muy alto. Estos resultados podrían diferir bastante de la situación de otras ciudades con mayores niveles de marginación. Por ejemplo, la estimación de este mismo índice en la ciudad de Oaxaca muestra una situación opuesta, con poco más de la mitad de sus AGEB (54.9\%) en condiciones de alta y muy alta marginación (CONAPO, 2010).

El IMU resultó mucho más claro en la identificación del lado opuesto del espectro, es decir, las diferencias espaciales que marcaron los grupos de mayor privilegio. Si por un lado la TBM por ECNT correspondió al proceso de segregación residencial del adulto mayor, la distribución espacial de la TMA evidenció la conformación de dos amplias "zonas protegidas" ante la mortalidad por cualquiera de los tres grupos de causas de muerte, como resultado de la autosegregación residencial que ejercen los grupos más aventajados (Tammaru, Marcińczak, Aunap, van Ham y Janssen, 2019, p. 4). Futuros estudios deben considerar 
las características que hacen de estas dos zonas un referente de salud a aspirar para el resto de la ciudad.

Por último, si bien las asociaciones más significativas de las desigualdades socioeconómicas en la mortalidad suelen encontrarse en ciudades de mayor tamaño (Borrell, Marí-Dell’Olmo, Serral, Martínez-Beneito y Gotsens, 2010, p. 709), la débil autocorrelación global entre la mortalidad por los tres grupos y el IMU en Hermosillo podría indicar la necesidad de explorar otros parámetros para medir las desigualdades socioeconómicas y de salud en la ciudad. En ciudades ubicadas en países de ingresos bajos-medios, las principales desigualdades en salud entre ricos y pobres tienen que ver con la mortalidad materna, infantil e incluso por ECNT, así como con enfermedades infecciosas, como la tuberculosis y VIH. Por su parte, los países de ingresos medios-altos suelen mostrar mayores disparidades socioeconómicas asociadas a conductas de riesgo, diabetes y enfermedades de trasmisión sexual (Brisbois et al., 2019, p. 77).

Desde el punto de vista de la enfermedad, la incorporación del componente de la morbilidad a la evaluación intraurbana resulta una tarea mucho más compleja en términos metodológicos, pero de mucha utilidad para una ciudad como Hermosillo. Desde la perspectiva de los determinantes, se sugiere explorar otro tipo de indicadores urbanos y del ambiente construido, asociados a la salud y a la calidad de vida en las ciudades. Indicadores de acceso a la salud, acceso a comida saludable, distribución de áreas verdes, espacios para la actividad física, "caminabilidad", movilidad y trasporte, exposición a temperaturas extremas, entre otros, contribuirán a un mejor entendimiento de los impactos del entorno urbano sobre los estados de salud y enfermedad (Navarro y Vélez, 2019; Pérez y Valencia, 2016; World Health Organization [WHO], 2016; Zuniga-Teran, Stoker, Gimbelett, Orr, Marsh, Guertin y Chalfoun, 2019).

\section{Limitaciones}

El análisis realizado sólo se enfocó en la mortalidad. La inclusión de información sobre morbilidad, componente esencial para un panorama epidemiológico completo, podría llevar a resultados diferentes a los aquí presentados. La agregación de defunciones por AGEB no permite establecer conclusiones respecto a las condiciones socioeconómicas de los residentes a escala individual. Por último, los resultados obtenidos sólo pueden interpretarse desde el criterio de georreferenciación utilizado, es decir, el lugar de residencia, hecho que afecta en mayor medida a aquellas defunciones relacionadas con la movilidad y la exposición en lugares de trabajo.

\section{Conclusiones}

El análisis de carga de la mortalidad mostró el predominio actual de las muertes asociadas a ECNT en la ciudad de Hermosillo. Atender las necesidades crecientes de la población afectada por estas enfermedades resulta de especial interés en la localidad. El análisis de la mortalidad bruta mostró la 
fuerte dependencia espacial de la mortalidad por ECNT y el envejecimiento poblacional como resultado de la segregación residencial del adulto mayor observada en el centro de la ciudad. Con el fin de focalizar las intervenciones para mejorar el acceso a la salud y el entorno construido de este segmento poblacional, los tomadores de decisiones deben considerar las implicaciones de dicho fenómeno.

Por otra parte, el análisis espacial de la mortalidad ajustada por edad evidenció espacios de fragmentación y de desigualdad en la ciudad. Estas desigualdades se refirieron en menor medida a zonas específicas de la periferia, cuyas condiciones de alta marginación las vuelven vulnerables ante la muerte relacionada con los tres grandes grupos de causas que requieren atención focalizada. Las principales desigualdades se observaron en la autosegregación espacial ejercida por los residentes agrupados en zonas cuya condición de muy baja marginación brindó un factor protector ante la muerte por cualquier causa con respecto al resto de la población.

El presente estudio buscó dar un panorama general actual de la mortalidad en su asociación con el envejecimiento y la marginación a una escala poco estudiada en México, debido en parte a las dificultades metodológicas del manejo e integración de información a escala intraurbana (Diez-Roux, 2015, p. s22). Desde nuestro conocimiento, éste es el primer estudio en México que mapea el total de muertes en una ciudad (agrupada por principales causas) a escala de AGEB. En ese sentido, se considera un ejercicio importante. Sin embargo, la complejidad y los problemas de salud del entorno urbano vuelven necesario ampliar su alcance. El uso de encuestas representativas, aunado a mejoras en los sistemas de vigilancia en lo que respecta a la georreferenciación de la morbilidad a pequeña escala y su capacidad de integración con fuentes de información relativas al entorno urbano, pueden ayudar a este propósito.

\section{Agradecimentos}

Agradezco a la Secretaría de Salud del Estado de Sonora por facilitar el acceso a la información y al Programa para el Desarrollo Profesional Docente (PRODEP) por los recursos otorgados para la realización de esta investigación.

\section{Referencias}

Agyei-Mensah, S., y de-Graft Aikins, A. (2010). Epidemiological transition and the double burden of disease in Accra, Ghana. Journal of Urban Health, 87(5), 879-897. doi: 10.1007/s11524-010-9492-y 
Álvarez de la Torre, G. (2017). Morfología y estructura urbana en las ciudades medias mexicanas. región y sociedad, 29(68), 153-191. doi: 10.22198/ rys.2017.68.a872

Álvarez, G., Lara, F., Harlow, S. D., y Denman, C. (2009). Mortalidad infantil y marginación urbana: análisis espacial de su relación en una ciudad de tamaño medio del noroeste mexicano. Revista Panamericana de Salud Pública, 26(1), 31-38.

Anselin, L. (1995). Local indicators of spatial association -LISA. Geographical Analysis, 27(2), 93-115. doi: 10.1111/j.1538-4632.1995.tb00338.x

Anselin, L. (1996). The Moran scatterplot as an ESDA tool to assess local instability in spatial association. En M. Fischer, H. J. Scholten y D. Unwin (eds.), Spatial Analytical Perspectives on GIS (pp. 111-125). Londres: Taylor \& Francis.

Besag, J., York, J., y Mollié, A. (1991). Bayesian image restoration, with two applications in spatial statistics. Annals of the Institute of Statistical Mathematics, 43(1), 1-20. doi: 10.1007/BF00116466

Borrell, C., Marí-Dell'Olmo, M., Serral, G., Martínez-Beneito, M., y Gotsens, M. (2010). Inequalities in mortality in small areas of eleven Spanish cities (the multicenter MEDEA project). Health \& Place, 16(4), 703-711. doi: 10.1016/j. healthplace.2010.03.002

Brisbois, B., O'Campo, P., y Kamalanathan, J. (2019). Inequities in cities and in urban health. En S. Galea, C. K. Ettman y D. Vlahov (eds.), Urban Health (pp. 76-84). Nueva York: Oxford University Press.

Cagney, K. A. (2019). Aging populations. En S. Galea, C. K. Ettman y D. Vlahov (eds.), Urban Health (pp. 59-69). Oxford; Nueva York: Oxford University Press.

Castro Luque, A. L. (2015). Travesías azarosas: relato demográfico del siglo XX sonorense. Hermosillo: El Colegio de Sonora.

Consejo Nacional de Evaluación de la Política de Desarrollo Social (CONEVAL). (2010). Evolución y determinantes de la pobreza de las principales ciudades de México, 1990-2010. Recuperado de https://www.coneval.org.mx/Informes/Pobreza/Pobreza\%20urbana/Evolucion_determinantes_de_la_pobreza_ urbana.pdf

Consejo Nacional de Población (CONAPO). (2010). Índice de marginación urbana 2010. Recuperado de http://www.conapo.gob.mx/en/CONAPO/Indice_de_ marginacion_urbana_2010

Diez-Roux, A. V. (2003). Residential environments and cardiovascular risk. Journal of Urban Health: Bulletin of the New York Academy of Medicine, 80(4), 569-589. doi: 10.1093/jurban/jtg065

Diez-Roux, A. V. (2015). Health in cities: is a systems approach needed? Cadernos de Saúde Pública, 31, 9-13. doi: 10.1590/0102-311XDE01S115

Dirección General de Información en Salud (DGIS-SS). (2017). Cubos dinámicos. Defunciones 1979-2015. Recuperado de http://www.dgis.salud.gob.mx/contenidos/basesdedatos/bdc_defunciones_gobmx.html

Frenk, J., Bobadilla, J. L., Stern, C., Frejka, T., y Lozano, R. (1991). Elementos para una teoría de la transición en salud. Salud Pública de México, 33(5), 448-462. 
Frenk, J., Lozano, R. , y Bobadilla, J. L. (1994). La transición epidemiológica en América Latina. Notas de Población, 60, 79-101.

Garrocho, C., y Campos-Alanis, J. (2015). Segregación residencial de los adultos mayores: relevancia del tema para México, marco teórico y políticas urbanas. En Segregación urbana y espacios de exclusión: ejemplos de México y América Latina (pp. 103-137). México: Universidad Nacional Autónoma de México, Instituto de Geografía y M. A. Porrúa.

Garrocho, C., y Campos-Alanis, J. (2016). Segregación socioespacial de la población mayor: la dimensión desconocida del envejecimiento. Zinacantepec: El Colegio Mexiquense.

Institute of Health Metrics and Evaluation (IHME). (2017). Global burden of disease (GBD). Recuperado de http:/ / www.healthdata.org/gbd

Instituto Nacional de Estadística y Geografía (INEGI). (2010a). Serie histórica censal e intercensal (1990-2010). Recuperado de http://www.beta.inegi.org. $\mathrm{mx} / \mathrm{proyectos} / \mathrm{ccpv} / \mathrm{cpvsh} /$ default.html

Instituto Nacional de Estadística y Geografía (INEGI). (2010b). Sistema para la consulta de información censal (SCINCE versión 05/2012). Recuperado de http: / / www.inegi.org.mx/est/scince/scince2010.aspx

Instituto Nacional de Estadística y Geografía (INEGI). (2014). Encuesta de cohesión social para la prevención de la violencia y la delincuencia (ECOPRED) 2014. Recuperado de http://www.beta.inegi.org.mx/programas/ecopred/2014/

Instituto Nacional de Estadística y Geografía (INEGI). (2015). Encuesta intercensal 2015. Recuperado de http://www.beta.inegi.org.mx/proyectos/enchogares/especiales/intercensal/

Kroll, M., Bharucha, E., y Kraas, F. (2014). Does rapid urbanization aggravate health disparities? Reflections on the epidemiological transition in Pune, India. Global Health Action, 7. doi: 10.3402/gha.v7.23447

Lara-Valencia, F., Álvarez-Hernández, G., Harlow, S. D., Denman, C., y García-Pérez, H. (2012). Vulnerabilidad socioambiental y mortalidad infantil en barrios de Hermosillo, Sonora. Salud Pública de México, 54(4), 367-374.

Lerch, M. , Oris, M. , y Wannar, P. (2017). Periurbanization and the transformation of the urban mortality gradient in Switzerland. Population: English Edition, 72(1), 93-122. doi: 10.3917/pope.1701.0093

Martinez, R., Soliz, P., Caixeta, R., y Ordunez, P. (2019). Reflection on modern methods: years of life lost due to premature mortality -a versatile and comprehensive measure for monitoring non-communicable disease mortality. International Journal of Epidemiology. doi: 10.1093/ije/dyy254

Massa, K. H. C., Pabayo, R., Lebrão, M. L., y Filho, A. D. P. C. (2016). Environmental factors and cardiovascular diseases: The association of income inequality and green spaces in elderly residents of São Paulo, Brazil. BMJ Open, 6(9), e011850. doi: 10.1136/bmjopen-2016-011850

Moore, E. G., y Pacey, M. A. (2004). Geographic dimensions of aging in Canada, 1991-2001. Canadian Journal on Aging / La Revue Canadienne Du Vieillissement, 23(5), S5-S21. doi: 10.1353/cja.2005.0037 
Murray, A. T., y Grubesic, A. (2015). Locational planning of health care facilities. En P. Kanaroglou, E. Delmelle, y A. Páez (eds.), Spatial Analysis in Health Geography (pp. 243-259). Surrey; Burlington: Ashgate Publishing Limited.

Navarro, A. C., y Vélez, M.-I. O. (2019). Obesogenic environment case study from a food and nutrition security perspective: Hermosillo City. International Journal of Environmental Research and Public Health, 16(3), 407. doi: 10.3390/ijerph16030407

Omran, A. R. (2005). The epidemiologic transition: a theory of the epidemiology of population change. The Milbank Quarterly, 83(4), 731-757. doi: 10.1111/j.1468-0009.2005.00398.x

Pérez, H. G., y Valencia, F. L. (2016). Equidad en la provisión de espacios públicos abiertos: accesibilidad, percepción y uso entre mujeres de Hermosillo, Sonora. Sociedad y Ambiente (10), 28-56.

Robson, B., Purdie, G., Cram, F., y Simmonds, S. (2007). Age standardisation -an indigenous standard? Emerging Themes in Epidemiology, 4(3). doi: 10.1186/1742-7622-4-3

Snyder, R., Rajan, J., Costa, F., Lima, H., Calcagno, J., Couto, R., Riley, L., Reis, M., Ko, A., y Ribeiro, G. (2017). Differences in the prevalence of non-communicable disease between slum dwellers and the general population in a large urban area in Brazil. Tropical Medicine and Infectious Disease, 2(3), 47. doi: 10.3390/tropicalmed2030047

Tammaru, T. , Marcińczak, S. , Aunap, R., van Ham, M. , y Janssen, H. (2019). Relationship between income inequality and residential segregation of socioeconomic groups. Regional Studies, 1-12. doi: 10.1080/00343404.2018.1540035

Tani, Y., Suzuki, N., Fujiwara, T., Hanazato, M., Kondo, N., Miyaguni, Y., y Kondo, K. (2018). Neighborhood food environment and mortality among older Japanese adults: results from the JAGES cohort study. International Journal of Behavioral Nutrition and Physical Activity, 15(1), 101. doi: 10.1186/ s12966-018-0732-y

Wang, D., Lau, K. K.-L., Yu, R., Wong, S. Y. S., Kwok, T. T. Y., y Woo, J. (2017). Neighbouring green space and mortality in community-dwelling elderly Hong Kong Chinese: a cohort study. BMJ Open, 7(7). doi: 10.1136/bmjopen-2016-015794

World Health Organization (WHO). (2016). What municipal authorities, local governments and ministries responsible for urban planning need to know. Recuperado de https://apps.who.int/iris/bitstream/handle/10665/250228/ WHO-NMH-NMA-16.89-eng.pdf; jsessionid=E161C3F2FA84D70219D8F89058ECO70E? sequence $=1$

Zuniga-Teran, A. A., Stoker, P., Gimblett, R. H., Orr, B. J., Marsh, S. E., Guertin, D. P. , y Chalfoun, N. V. (2019). Exploring the influence of neighborhood walkability on the frequency of use of greenspace. Landscape and Urban Planning, 190, 103609. doi: 10.1016/j.landurbplan.2019.103609 